\title{
A New Method for Numerical Conformal Mapping of Doubly-connected Domain
}

\author{
Fuming Lai ${ }^{1, a}$, Yibin Lu ${ }^{1, b^{*}}$,Yingzi Wang ${ }^{2, c}$, and Dean $W^{3, d}$ \\ ${ }^{1}$ Faculty of Science, Kunming University of Science and Technology, Kunming 650500, China; \\ ${ }^{2}$ Computer Center, Kunming University of Science and Technology, Kunming 650500, China; \\ ${ }^{3}$ School of Mathematical Sciences, University of Electronic Science and Technology,Chengdu \\ 611731, China.
}

aFuming7710@163.com, ${ }^{\mathrm{b}}$ luyibin@kmust.edu.cn, ${ }^{\mathrm{T}}$ wyingzi@kmust.edu.cn, dwudean_cn@hotmail.com

\begin{abstract}
Keywords: numerical conformal mapping, doubly-connected domain, charge simulation method, the iteration method by correcting characteristic values.

Abstract. Conformal mapping is an important issue of complex function theory. In this paper, a new method for numerical conformal mapping of doubly-connected domain is considered. This method calculates the constraint equations of numerical conformal mapping based on charge simulation method of doubly-connected domain using the improvement of iteration method by correcting characteristic values, and the approximate conformal mapping function is constructed using new charge points and conformal mapping radius. We illustrate the efficiency of the proposed method by some numerical results.
\end{abstract}

\section{Introduction}

Conformal mapping has many practical use, such as fluid flow, image processing, elec-tromagnetics, and other fields. Conformal mapping method includes analytical and numerical calculation method. The theoretical basis of analytical method is Riemann Theorem, it merely proves the existence of transformation function, but can not obtain a specific function expression. So, for many of the practical problems must use numerical calculation method to calculate conformal mapping. Several numerical calculation methods have been proposed for calculating numerical conformal mapping, they include integral equation method [1-3], orthonormalization method [4], charge simulation method [5-7], and so on. The charge simulation methods for numerical conformal mapping were proposed by a Japanese mathematician K.Amano. However, he did not give a specific algorithm for solving the constraint equations.

In this paper, improvement of the iteration method by correcting characteristic values for numerical conformal mapping of doubly-connected domain is considered. With this algorithm, the constraint equations of doubly-connected domain is solved, and the approximate conformal mapping function is constructed using the charge points and conformal mapping radius. Finally, the efficiency of the proposed method is illustrated by several numerical examples .

\section{Numerical Conformal Mapping Calculation Method Based On Charge Simulation Method}

For this part, the method for numerical conformal mapping of doubly-connected domain is give [7]. A finite doubly-connected domain $D$ bounded by two closed Jordan curves $C_{1}$ and $C_{2}$ in the $z$-plane mapped onto a circular annulus $\mu<|w|<1$ in the $w$-plane. The boundary $C=C_{1} \cup C_{2}$ consisted of the exterior $C_{1}$ and the interior $C_{2}, C_{1}$ and $C_{2}$ are mapped onto the concentric circles $|\omega|=1$ and $|\omega|=w$ (fig.1) [8-9]. Assume, without loss of generality, the origin $z=0$ lies in $D$ and $f(0)=0$.Then the mapping function $f(z)$ is unique under the normalizing condition 


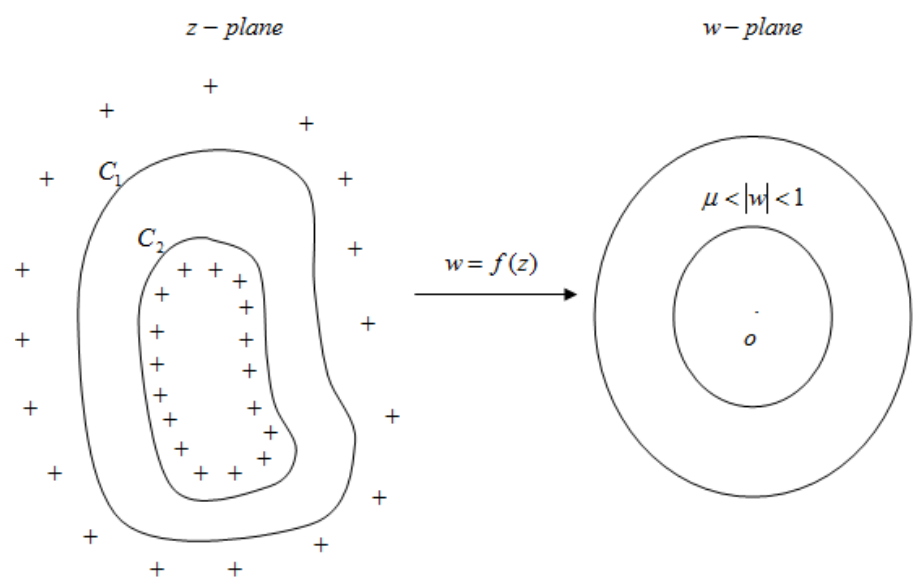

Fig. 1 Conformal mapping of doubly-connected domain $f(0)=0$ and $f^{\prime}(0)>0$, which can be expressed as

$$
f(z)=z \exp (g(z)+i h(z)), z \in \bar{D}
$$

here $\bar{D}=D+C_{1}+C_{2} . g(z)$ is the solution of the Dirichlet problem

$$
\begin{cases}\nabla^{2} g(z)=0, & z \in D \\ g(z)=-\log |z|, & z \in C_{1} \\ g(z)=\log \mu-\log |z|, & z \in C_{2}\end{cases}
$$

and, the function $h(z)$ and $g(z)$ are conjugate harmonic in $D$.

Based on charge simulation method, $h(z)$ and $g(z)$ can be approximated by

$$
\begin{aligned}
& G(z)=-\sum_{i=1}^{N} Q_{i} \log \left|z-\zeta_{i}\right|, \quad z \in \bar{D} \\
& H(z)=-\sum_{i=1}^{N} Q_{i} \arg \left(z-\zeta_{i}\right), \quad z \in \bar{D}
\end{aligned}
$$

the charge points $\zeta_{i}(i=1,2, \mathrm{~L}, N)$ are arranged outside the given domain $D$. More precisely, $N_{1}+N_{2}=N, N_{1}$ and $N_{2}$ charge points are arranged outside the domain bounded by $C_{1}$ and inside the domain bounded by $C_{2}$ [9]. The $N$ charges $Q_{i}$ can be determined to satisfy the Dirichlet boundary condition at $N$ collection points $z_{j}$ arranged on the boundary $C_{1}$ and $C_{2}$, that is to say,

$$
\begin{aligned}
& \sum_{i=1}^{N} Q_{i} \log \left|z_{j}-\zeta_{i}\right|=\log \left|z_{j}\right|, \quad z_{j} \in C_{1} \\
& \sum_{i=1}^{N} Q_{i} \log \left|z_{j}-\zeta_{i}\right|+\log M=\log \left|z_{j}\right|, \quad z_{j} \in C_{2} \\
& \sum_{i=1}^{N_{2}} Q_{i}=0
\end{aligned}
$$

By Eq. 5-Eq.7, a linear equations has the unknown of $Q_{i}(i=1,2, \mathrm{~L}, N)$ and $\log M$ can be given,

$$
\left[\begin{array}{ccccccc}
a_{11} & \mathrm{~L} & a_{1, N_{2}} & a_{1, N_{2}+1} & \mathrm{~L} & a_{1 N} & 0 \\
\mathrm{M} & \mathrm{O} & \mathrm{M} & \mathrm{M} & \mathrm{L} & \mathrm{M} & \mathrm{M} \\
a_{N_{1} 1} & \mathrm{~L} & a_{N_{1}, N_{2}} & a_{N_{1}, N_{2}+1} & \mathrm{~L} & a_{N_{1}, N} & 0 \\
a_{N_{1}+1,1} & \mathrm{~L} & a_{N_{1}+1, N_{2}} & a_{N_{1}+1, N_{2}+1} & \mathrm{~L} & a_{N_{1}+1, N} & 1 \\
\mathrm{M} & \mathrm{M} & \mathrm{M} & \mathrm{M} & \mathrm{O} & \mathrm{M} & \mathrm{M} \\
a_{N 1} & \mathrm{~L} & a_{N, N_{2}} & a_{N, N_{2}+1} & \mathrm{~L} & a_{N N} & 1 \\
0 & \mathrm{~L} & 0 & 1 & \mathrm{~L} & 1 & 0
\end{array}\right]\left[\begin{array}{c}
Q_{1} \\
\mathrm{M} \\
Q_{N_{1}} \\
Q_{N_{1}+1} \\
\mathrm{M} \\
Q_{N} \\
\log M
\end{array}\right]=\left[\begin{array}{c}
\log \left|z_{1}\right| \\
\mathrm{M} \\
\log \left|z_{N_{1}}\right| \\
\log \left|z_{N_{1}+1}\right| \\
\mathrm{M} \\
\log \left|z_{N}\right| \\
0
\end{array}\right]
$$


where, $a_{i j}=\log \left|z_{j}-\zeta_{i}\right|$. Finally, numerical conformal mapping of doubly-connected is calculated by $z_{i}, \zeta_{i}, Q_{i}$ and $M[9-10]$.

\section{Improvement of the Iteration Method by Correcting Characteristic Values}

The Eq. 8 is called constraint equations[7], it can be written in the form of standard linear equations

$$
A x=b,
$$

where $A \in R^{(N+1) \times(N+1)}, x \in R^{N+1}$, and $b \in R^{N+1} . N$ is the number of charge simulation points. Matrix A is ill-conditioned that a small perturbation of $b$ will lead to a large perturbation of the solution.

According to the principle of least squares problem [11-12], the normal equation can be obtained

$$
A^{T} P A x=A^{T} P b,
$$

where $A^{T} P A$ is the symmetric positive definite. Obviously $x=\left(A^{T} P A\right)^{-1} A^{T} P A$, however, the approach is not feasible because $x=\left(A^{T} P A\right)^{-1}$ is very ill-conditioned that inverse will be unstable for $A^{T} P A$. Using this method is difficult to obtain a good results.

A number of numerical methods have been proposed for solving ill-conditioned problem, such as Gram-Schmidt method, ridge estimation [11], singular value decomposition technique(SVD)[13], the iteration method by correcting characteristic values [14-15], etc. proposed using to solve equation (3.1). Assumed $\mathrm{E}$ is the $N \times N$ identity matrix, the (3.2) on both sides plus $x$, then it can be written as

$$
\left(A^{T} P A+E_{N}\right) x=A^{T} P b+x .
$$

Obviously, $A^{T} P A+E_{N}$ is symmetric positive definite. we can obtain the iteration formula

$$
x^{k}=\left(A^{T} P A+E_{N}\right)^{-1}\left(A^{T} P b+x^{k-1}\right),(k=1,2, \mathrm{~L})
$$

The spectral radius of $\left(A^{T} P A+E_{N}\right)^{-1}$ is $\frac{1}{1+\sigma}, \sigma$ is the smallest eigenvalues of $A^{T} P A$. Since $\frac{1}{1+\sigma}$ is close to 1 , the above method converges to the exact solution is too slow, and the solution is not accurate. In order to improve the convergence rate and the accuracy of solutions, the Eq. 10 on both sides plus $\mu x$, for $\mu$ is correction factor [16-17]. Then, the improvement of iteration method by correcting characteristic values formula is given by

$$
\left(A^{T} P A+\mu E_{N}\right) x=A^{T} P b+\mu x .
$$

then we know its iterative formula

$$
x^{k}=\left(A^{T} P A+\mu E_{N}\right)^{-1}\left(A^{T} P b+\mu x^{k-1}\right) \cdot(k=1,2, \mathrm{~L})
$$

The spectral radius of $\mu\left(A^{T} P A+\mu E_{N}\right)^{-1}$ is $\frac{\mu}{\mu+\sigma}, \frac{\mu}{\mu+\sigma}<\frac{1}{1+\sigma}$. Thus, simply select the appropriate correction factor $\mu$, the new spectral radius can be less than 1 , and the convergence rate will be accelerated.

\section{Numerical Experiments}

In this section, numerical experiments are given to illustrate the performance of our proposed methods. The experiments were performed under MATLAB 7.0. The error formula of numerical

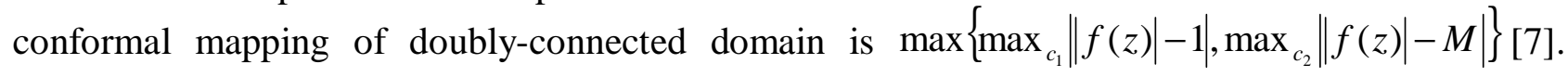
$P=E, E$ is the identity matrix. Using two ellipses $C_{1}: \frac{x^{2}}{a_{1}^{2}}+\frac{y^{2}}{b_{1}^{2}}=1, C_{2}: \frac{x^{2}}{a_{2}^{2}}+\frac{y^{2}}{b_{2}^{2}}=1$ as a boundary .

Example $a_{1}=7, b_{1}=5, a_{2}=5, b_{2}=1$. 
The stopping criterion of the methods is that the error should satisfy $\left\|x-x_{\text {new }}\right\| /\|x\|>10^{-3}$ or the number of iterations is larger than 400 , The correction factor is $\mu=0.002$.

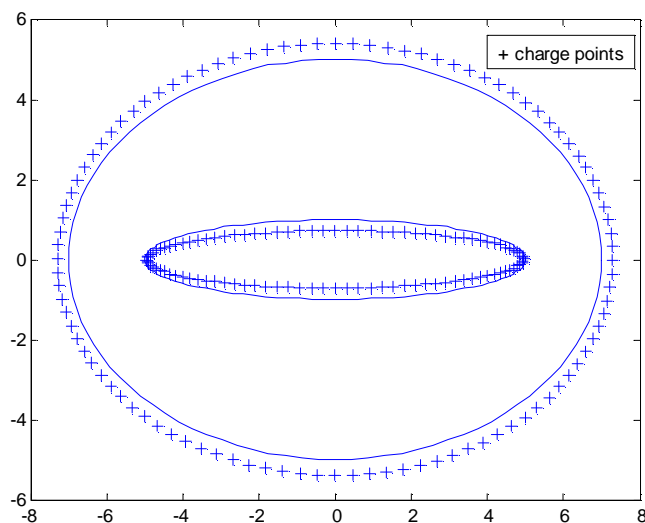

(a)

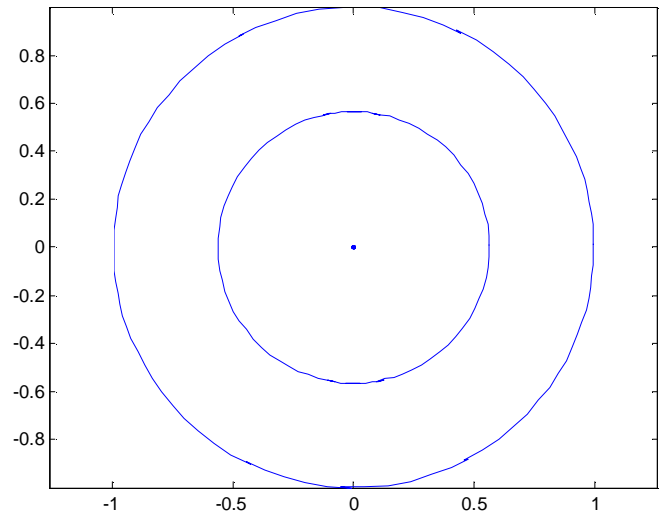

(b)

Fig. 2 Arrangement of charge points in the charge simulation method

Figure 1(a) shows the distribution of $N=200$ charge simulation points, Of which $N_{1}=100$ located in the exterior $C_{1}: \frac{x^{2}}{7^{2}}+\frac{y^{2}}{5^{2}}=1$, and the other $N_{2}=100$ are located in the interior $C_{2}: \frac{x^{2}}{5^{2}}+y^{2}=1$. Mapping function $f(z)$ maps $C_{1}$ and $C_{2}$ onto the concentric circles shows in Figure 1(b).

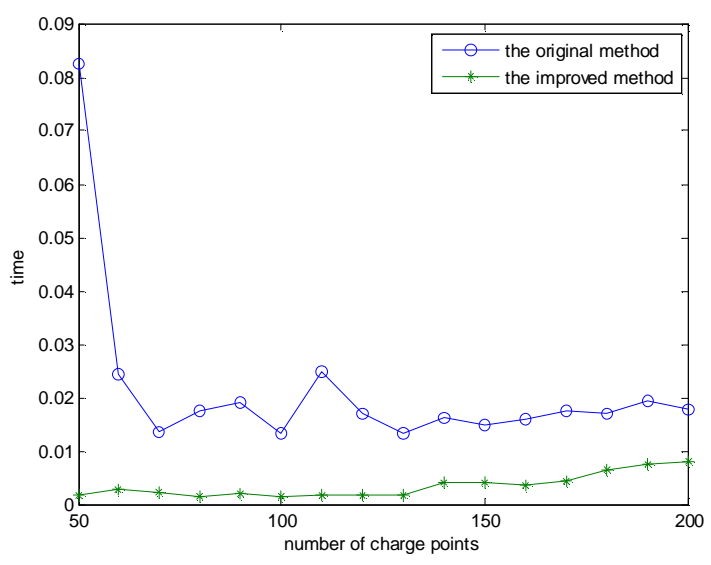

(a)

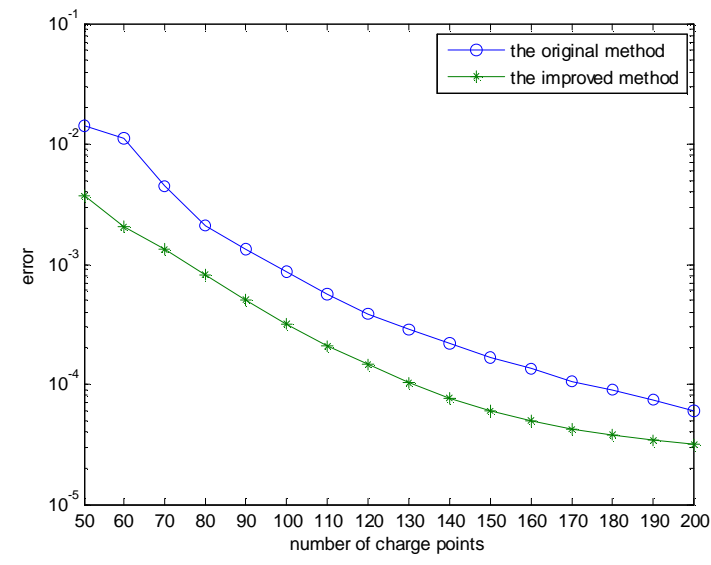

(b)

Fig. 3 Program run times and iterations

As can be seen from Figure 3(a), the improved method running less time than the iteration method by correcting characteristic values. From Figure 3(b), with the increase of the charge simulation points, the error getting smaller and smaller, and the improved method obtained higher accuracy than the original method.

For further verify the the feasibility of algorithm previously proposed, making contour lines on region $D$ bounded by curves $C_{1}$ and $C_{2}$. The contour lines and the image is mapped are shown in Figure 4(a) and Figure 4(b) respectively. Blue thick solid lines in Figure 4(a) are the boundaries $C_{1}$ and $C_{2}$ of region $D$, the thin blue lines is the contour lines. As can be seen from Figure 4(b), Boundaries are mapped onto the concentric circles, the interior region of $D$ is still mapped into the interior region of mapped imagine, this verifies the validity of the algorithm . 


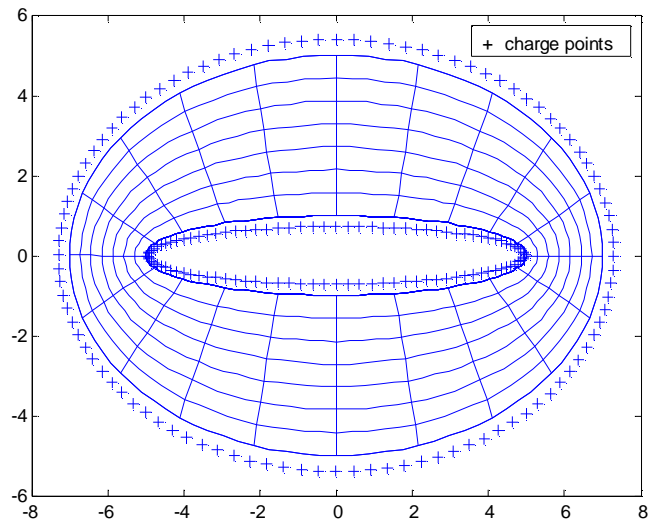

(a)

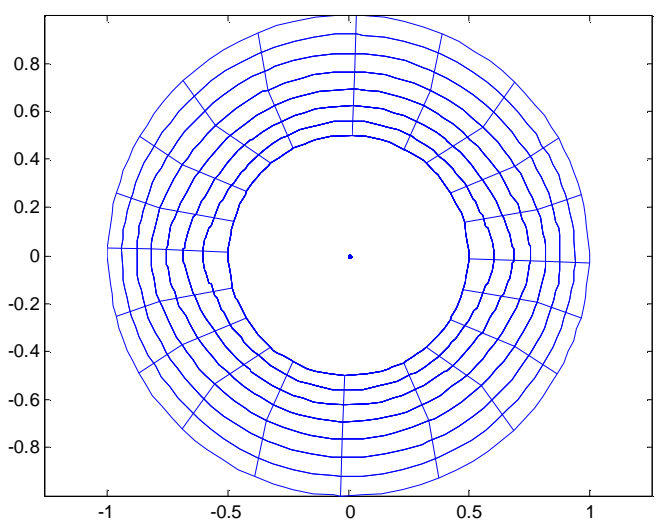

(b)

Fig. 4 The contour lines and the image is mapped

\section{Conclusion}

In this paper, a new method has been proposed for improving the accuracy and the speed of numerical conformal mapping of doubly-connected domain. The applicability of our method has been demonstrated with numerical results. The accuracy and the speed of conformal mapping by the proposed method is better than achievable by the charge simulation method. The results of numerical conformal mapping of doubly-connected domain is simulated using the contour lines. This method is considered for computing conformal mapping of multiply-connected domains in the future.

\section{References}

[1] G.T. Symm, An integral equation method in conformal mapping, J. Numerische Mathematik. 9 (1966) 250-258.

[2] A.W.K. Sangawia , A.H.M Murida, M.M.S Nasserd, Linear integral equations for conformal mapping of bounded multiply connected regions onto a disk with circular slits, J. Applied Mathematics and Computation. 218 (2011) 2055-2068.

[3] G.T. Symm, Conformal mapping of doubly-connected domains, J. Numerische Mathematik. 13 (1969) 448-457.

[4] P. Rabinowitz, Numerical experiments in conformal mapping by the method of orthonormal polynomials, J. ACM. 13(1996) 296-303.

[5] K. Amano, Numerical conformal mappings of exterior domains based on the charge simulation method, J. Trans Inform Process Soc Japan. 29 (1998) 62-72(in Japanese).

[6] K. Amano, Numerical conformal mappings of interior domains based on the charge simulation method, J. Trans Inform Process Soc Japan. 29 (1988) 697-699(in Japanese).

[7] K. Amano, Numerical conformal mapping of doubly-connected domains based on the charge simulation method, J.Trans Inform Process Soc Japan. 29 (1988) 914-919(in Japanese).

[8] K. Amano, A bidirectional method for numerical conformal mapping based on the charge simulation method, J. Trans Inform Process Soc Japan. 28 (1991) 473-482(in Japanese).

[9] K. Amano, A charge simulation method for numerical conformal mapping of interior,exterior and doubly-connected domains, J. Journal of Computational and Applied Mathematics. 53 (1994) 354-361.(in Japanese)

[10] Y. Lu, D. Wu, Y. Wang, et al, The accuracy improvement of numerical conformal mapping using the modified Gram-Schmidt method, C.The 19th International Conference on Industrial Engineering and Engineering Management. Springer Berlin Heidelberg. (2013) 555-56.

[11] G.H. Golub, C.F. Van Loan, Matrix Computation, 4rd ed., Posts \& Telecom Pres, Beijing, 2014. [12] N. Malyshev, A unified theory of conditioning for linear least squares and Tikhonov regularization solutions, J. SIAM Journal on Matrix Analysis \& Applications. 20(2003) 1186-1196. 
[13] G.W. Stewart, On the Early History of the Singular Value Decomposition, J. SIAM Review. 35(1993): 551-566.

[14] X. WANG, D. LIU, Iteration method by correcting characteristic value for ill-conditioned equations in the least square estimations, J. Journal of Hubei Znstitute for Nationalities. 20(2002) 1-4. [15] X. WANG, The method of improving least square estimate in unbiased estimation class, J. Journal of Wuhan Technical University of Surveying and Mapping. 20(1995) 46-50.

[16] D. HUANG, J. SHI, et al, Iterative method based on the morbid equation, J. Science of Surveying and Mapping. 38(2013) 35-37

[17] G.H. Golub , P.C. Hansen, D.P. O'leary, Tikhonov regularization and total least square, J. SIAM Journal on Matrix Analysis and Applications. 21(1999) 185-194. 